\title{
Frecuencia de ansiedad preoperatoria y factores asociados en gestantes programadas a cesárea
}

\author{
Freddy Quintana-Guardo', Álvaro Monterrosa-Castro², Teresa Beltrán-Barrios ${ }^{3}$
}

\section{RESUMEN}

Objetivo: estimar la frecuencia de la ansiedad preoperatoria (AP) y los factores asociados en las gestantes programadas con cesárea electiva.

\begin{abstract}
Métodos: estudio transversal en mujeres con embarazo de término sin actividad uterina ni complicaciones médicas, quirúrogicas u obstétricas, realizado previo a la intervención de cesárea electiva, en Cartagena, Colombia. Participación anónima y voluntaria con consentimiento informado. Se aplicó un formulario de características sociodemográficas y la versión en español de la escala Amsterdam Preoperative Anxiety and Information Scale (APAIS). Se realizó regresión logística no ajustada para observar la asociación de las variables cualitativas con AP, se estimó el coeficiente de correlación entre las puntuaciones de las dos subescalas del APAIS con las variables cuantitativas.
\end{abstract}

Resultados: se estudiaron 326 embarazadas, con edad entre $28,5 \pm 6,4$ años; 7,9 \% adolescentes; $24,2 \%$ primigestantes; $81,9 \%$ con cesárea anterior y 70,8 \% sin otras cirugías previamente. El 45 \% informó no estar de ningún modo preocupada por la cirugía o anestesia y el 7,8 \% quería saber mucho más sobre el procedimiento. La frecuencia de AP y de la necesidad de tener mucha más información de la cirugía o anestesia fue del 9,2\%. El número de familiares en la sala de espera se correlacionó negativamente con AP, rho: -0,127 [IC 95 \%: -0,232-0,019]. La necesidad de mucha más información sobre la cirugía o anestesia se asoció con el aumento

1 Estudiante de Medicina. Integrante del Grupo de investigación Salud de la Mujer. Facultad de Medicina. Universidad de Cartagena. Colombia.

2 Médico. Especialista en ginecología y obstetricia. Profesor y director del Grupo de investigación Salud de la Mujer. Facultad de Medicina. Universidad de Cartagena. Colombia.

3 Filósofa. Candidata a Maestría en Filosofía. Integrante del Grupo de investigación Salud de la Mujer. Facultad de Medicina. Universidad de Cartagena. Colombia.

Correspondencia: Álvaro Monterrosa-Castro; alvaromonterrosa@gmail.com

Recibido: febrero 20 de 2019

Aceptado: mayo 27 de 2019

Cómo citar: Quintana-Guardo F, Monterrosa-Castro A, Beltrán-Barrios T. Frecuencia de ansiedad preoperatoria y factores asociados en gestantes programadas a cesárea. latreia. 2020 Ene-Mar;33(1):5-16. DOI 10.17533/udea.iatreia.31. 
en la frecuencia de AP, OR: 10,9 [IC $95 \%$ : 4,54-26,36]. También lo fueron los estados civiles: unión libre y separada, con respecto a casada: OR: 3,7 [IC 95 \%: 1,59,2] y OR: 4,4 [IC 95 \%: 1,0-19,3], respectivamente.

Conclusión: la frecuencia de AP previa a la cesárea fue de 9,2\%. La necesidad de tener más información sobre la cirugía o anestesia se asoció diez veces mayor la presencia de AP y el número de familiares en la sala de espera se correlacionó negativamente con AP, pero el tamaño del coeficiente de correlación se interpretó como despreciable.

\section{PALABRAS CLAVE}

Ansiedad; Cesárea; Cuestionario de Salud del Paciente; Periodo Preoperatorio

\section{SUMMARY}

Frequency of preoperative anxiety and associated factors in pregnant women scheduled to cesarean section

Objective: To estimate frequency of preoperative anxiety (PA) and associated factors in pregnant women scheduled for elective cesarean section.

Methods: Cross sectional study in full term pregnant women without uterine activity and without medical, surgical or obstetric complications. Was carried out before elective cesarean section in Cartagena, Colombia. Anonymous and voluntary participation with informed consent. A sociodemographic characteristic form and the Spanish version of the Amsterdam Preoperative Anxiety and Information Scale (APAIS) were applied. Unadjusted logistic regression was performed to observe the association of the qualitative variables with PA and the correlation coefficient between the scores of the two subscales of the APAIS and the quantitative variables was estimated.

Results: 326 pregnant women were studied. Age 28,5 $\pm 6,4$ years; $7,9 \%$ teenagers; $24,2 \%$ primigravid, $81,9 \%$ with previous cesarean section and $70,8 \%$ without other previous surgery. 45,0 \% reported not being in any way worried about surgery/anesthesia and $7,8 \%$ wanted to know much more about the procedure. The frequency of PA and the need of much more information about surgery/anesthesia was 9,2 \%. The number of relatives in the waiting room was negatively correlated with PA, rho: -0,127[CI $95 \%$ : -0,232 to $-0,019]$. The need of much more information about surgery/anesthesia was associated with increased frequency of PA, OR:10,9 [CI $95 \%$ : 4,54-26,36]. Also, civil status was associated: free union and separate respect to married, OR: 3,7[CI $95 \%$ : 1,5-9,2] and OR: 4,4[CI $95 \%$ : 1,0-19,3], respectively.

Conclusion: The frequency of PA before cesarean section was $9,2 \%$. The need of much more information about surgery/anesthesia, was associated ten times greater presence of PA and the number of relatives in the waiting room was negatively correlated with $\mathrm{AP}$, but the size of the correlation coefficient was interpreted as negligible.

\section{KEY WORDS}

Anxiety; Cesarean Section; Patient Health Questionnaire; Preoperative Period

\section{INTRODUCCIÓN}

La ansiedad puede ser una característica de la personalidad de un individuo, o puede ser un comportamiento causado por un evento pasajero e importante que se percibe consciente o inconscientemente como de riesgo. Al primer caso se le denomina ansiedadrasgo y al segundo, ansiedad-estado ${ }^{(1-3)}$. La ansiedadestado se suele expresar por disforia y contiene elementos somáticos y psicológicos. Los primeros son derivados de la estimulación del sistema nervioso vegetativo, con aumento del tono simpático. Los últimos son el resultado de la estimulación del sistema límbico y la corteza cerebral ${ }^{(1,4)}$.

La ansiedad preoperatoria (AP) es del tipo ansiedadestado, se presenta en los pacientes que van a ser sometidos a un procedimiento quirúrgico ${ }^{(5)}$. Se ha señalado una asociación entre AP con la necesidad de usar mayores dosis de fármacos anestésicos o analgésicos, mayor morbilidad o dolor posoperatorio, alargamiento en la estancia hospitalaria o prolongación en la recuperación ${ }^{(3,5)}$. Se ha propuesto para identificar la AP el Inventario de la Ansiedad Rasgo-Estado 
[IDARE], que es la versión en español del State Trait Anxiety Inventary [STATE], la Escala Visual Análoga [VAS] y Amsterdam Preoperative Anxiety And Information Scale [APAIS] ${ }^{(6,7)}$.

Uno de los procedimientos quirúrogicos más frecuentes es la cesárea, cuya tasa se ha incrementado en todos los países desarrollados y no desarrollados. En Colombia se pasó del $24,8 \%$ en 1998 al 46,1 \% en el $2013^{(8)}$. Ninguna región geográfica de Colombia cumple con el planteamiento de la OMS, que señala que no hay razón aloguna para que existan tasas de cesáreas superiores a $10-15 \%{ }^{(9)}$.

Chen et al., ${ }^{(10)}$ señalan que la cesárea está asociada con un mayor incremento en el riesgo de los síntomas asociados con el estrés, comparado con el parto vaginal. De otra parte, Wyatt et al., ${ }^{(11)}$ señalan que, en modelos de animales en embarazo, la presencia del estrés psicológico materno se puede acompañar con cambios desfavorables en la frecuencia cardiaca, presión sanguínea y oxigenación fetal; por lo que reducir la ansiedad materna puede mejorar las condiciones del bienestar intrauterino. Es necesario conocer la frecuencia de la $\mathrm{AP}$ antes de la cesárea en diferentes comunidades; no obstante, no se identificaron estudios en la población colombiana. El objetivo de esta investigación fue estimar la frecuencia de AP y los factores asociados en un grupo de gestantes antes de ser sometidas a cesárea, residentes en una ciudad del caribe colombiano.

\section{MÉTODOS}

Diseño: estudio de corte transversal que hace parte de la línea de investigación Gestación, declarada y avalada por la Universidad de Cartagena, Colombia.

Participantes: mujeres que estaban programadas electivamente con cesárea de cualquier edad, que no fuesen consumidoras de sustancias psicoactivas, que no tuviesen diagnóstico previo de psicopatologías, que no presentasen actividad uterina y no tuviesen complicaciones médicas, quirúrgicas u obstétricas al momento de la selección. Con embarazo entre 38-40 semanas, feto único, en cefálica, con actividad cardíaca presente $y$ monitoreo fetal reactivo realizado dentro de las seis horas previas. Las gestantes fueron identificadas en la sala preoperatoria de la Clínica Santa Cruz de Bocagrande, en Cartagena, Colombia, institución de salud de tercer nivel que atiende gestantes residentes en áreas urbanas o rurales de todos los niveles socioeconómicos. En el segundo semestre del año 2017 una auxiliar de enfermería previamente capacitada, brindó la información necesaria para que las mujeres realizaran sin apoyo el llenado del formulario y el consentimiento informado. Las gestantes fueron invitadas a participar diariamente $y$ de forma continua hasta completar el tamaño de la muestra estimada. No se realizó una selección aleatoria. Se excluyeron las mujeres que no desearon participar, las que no comprendieron la herramienta a utilizar y las que, por cualquier razón, debieron recibir anestesia general.

Herramientas: se utilizó un formulario que constaba de dos partes. La primera interrogaba sobre características sociodemográficas: edad, estado civil, actividad laboral, historia obstétrica y número de personas acompañantes en la sala de espera. La segunda era la versión en español de la escala Amsterdam Preoperative Anxiety and Information Scale (APAIS), que fue desarrollada en 1996 por Moermann et al., y está compuesta por seis ítems tipo Likert que se puntúan de 1-5, siendo 1: de ningún modo y, 5: mucho. El instrumento se divide en dos subescalas, las cuales se valoran por separado. Una evalúa la AP a la cirugía o anestesia [preguntas 1, 2, 4, 5], la cual se identifica si la puntuación es superior a 13. La otra, interpela sobre la necesidad de información sobre la cirugía o anestesia [preguntas 3, 6], una puntuación entre 2-4 indica poca o ninguna necesidad de más información, 5-7 la necesidad moderada de más información y 8-10 la necesidad de más información. Como no se identificaron traducciones en español que hubiesen sido validadas en gestantes o en mujeres colombianas, se utilizó la versión aplicada a la población española de ambos sexos y sometida a cirugía general. En la validación del constructo los autores obtuvieron Alpha de Cronbach de 0,84 ${ }^{(7)}$. Inmediatamente los formularios fueron regresados por las gestantes, la auxiliar de enfermería los revisó, identificó para cađa paciente la presencia de AP y los legajó así: los incompletos o inadecuadamente diligenciados en la carpeta Eliminados y, los adecuadamente llenados en la carpeta Adecuados. Semanalmente los entrego a los investigadores, quienes realizaron una segunda revisión.

Tamaño de la muestra: en el año 2016 en la Clínica Santa Cruz de Bocagrande se realizaron, según los libros 
de registro de cirugías, 2124 cesáreas electivas, y ese número es considerado el universo del estudio. Se calculó el tamaño de muestra de 326 gestantes por medio de la calculadora gratuita online Netquest, con heterogeneidad del $50 \%$, margen de error del $5 \%$ y nivel de confianza del 95 \%. La prevalencia esperada de la AP no se consideró entre los factores para el cálculo del tamaño de la muestra. El cálculo fue realizado para una encuesta a partir de la fórmula que se basa en el margen de error.

Ética: el estudio se realizó con la aprobación de la Gerencia y el Comité de ética de la Clínica Santa Cruz de Bocagrande. La participación fue anónima y voluntaria; todas las gestantes llenaron el consentimiento informado tal como lo establece la declaración de HeIsinki ${ }^{(12)}$. Se tuvo en cuenta las normas científicas, técnicas y administrativas para la investigación en salud establecidas en la Resolución 8430 de 1993 del Ministerio de Salud de la República de Colombia, lo que permite considerar el estudio como investigación de riesgo mínimo ${ }^{(13)}$.

Cuando la auxiliar de enfermería identificaba una puntuación superior a 13 en la subescala de ansiedad, se notifica al anestesiólogo a cargo de la paciente para que tome las acciones según el protocolo de atención.

Análisis estadístico: la información obtenida fue tabulada en una base de datos en Microsoft Excel ${ }^{\odot} 2016$. EI análisis estadístico se realizó con EPI-INFO-7 [Centers for Disease Control and Prevention, Atlanta, EE.Uu]. La variable cuantitativa edad, se presenta en media con desviación estándar, por haber tenido distribución paramétrica. La variable número de familiares en sala de espera en mediana con rango intercuartil (RI), por no ser paramétrica. Las variables: número de embarazos, número de abortos, número de partos y número de cesáreas fueron convertidas en cualitativas. Las variables cualitativas se expresan en valores absolutos, porcentuales e IC 95 \%. Se estimó el coeficiente de correlación de Spearman (rho) entre la puntuación de las dos subescalas del APAIS con las variables cuantitativas: edad, embarazos, abortos, partos, cesáreas, cirugías previas y número de familiares en sala de espera. El tamaño del coeficiente de correlación se interpretó de la siguiente manera. Seguimos la clasificación presente en la guía para el uso apropiado del coeficiente de correlación en investigación médica ${ }^{(14)}$ : ninguna correlación: 0,00, despreciable: 0,01-0,29, baja: 0,30-0,49, moderada:
0,50-0,69, alta: 0,70-0,89, muy alta: 0,90-0,99 y, perfecta: 1,00 , pudiendo ser positiva o negativa y estadísticamente significativa o no significativa. Se realizó regresión logística no ajustada para estimar la asociación entre la AP con la "necesidad de mucha más información sobre la cirugía/anestesia” y con las siguientes características sociodemográficas: actividad laboral fuera de casa, dificultad para quedar en embarazo, antecedente de complicaciones en el embarazo actual, antecedente de cirugía previa, antecedente de cesárea, antecedente de parto vaginal, antecedente de aborto, primigestante, edad superior a 40 años, edad entre 30-39 años, edad entre 20-29 años y adolescente. La asociación se expresa en OR con IC 95 \%. Se calculó el estadístico de confiabilidad Alpha de Cronbach para cada una de las dos subescalas, ya que ellas se evalúan por separado. Esto como parte de la validación del constructo en gestantes colombianas para ser sometidas a cesárea (datos no presentados). Un valor de $\mathrm{p}<0,05$ fue considerado estadísticamente sionificativo.

\section{RESULTADOS}

En total fueron invitadas a participar 369 gestantes de las cuales 15 (4\%) se rehusaron a participar, por lo tanto, fueron aplicados 354 formularios; de estos, 28 (7,9\%) tuvieron enmendaduras, estaban mal diligenciados o incompletos, por tal razón fueron eliminados. Quedan entonces 326 formularios de igual número de embarazadas que se consideraron para el análisis.

La edad media de las participantes fue de 28,5 $\pm 6,4$ años, con edad mínima de 13 y máxima 48. El 7,9\% eran adolescentes y el 5,5\% de las gestantes añosas. 267 (81,9 \%) mujeres fueron sometidas a cesárea por tener cesárea previa. Para las otras gestantes, las dos más frecuentes indicaciones para ser programadas a cesárea fueron desproporción cefalopélvica y presentación podálica. En su mayoría la totalidad de las estudiadas eran gestantes sanas, ninguna tenía morbilidad obstétrica o fetal. La Tabla 1 presenta las características sociodemográficas explorađas.

La puntuación promedio de la subescala de ansiedad fue $7,38 \pm 3,45$. La frecuencia de la AP fue 9,2 \%, ya que 30 mujeres tuvieron puntuación superior a 13 en la subescala de ansiedad. Igual proporción informaron tener necesidad de mucha más información sobre la 
cirugía o anestesia, mientras que $61(18,7 \%)$ pacientes la necesitaban moderadamente y $235(72,1 \%)$ pacientes tenían poca a ninguna necesidad de más información.
La puntuación promedio de la subescala de necesidad de información fue 3,91 $\pm 2,17$. La Tabla 2 presenta las respuestas a cada uno de los ítems de la escala APAIS.

Tabla 1. Características sociodemográficas en una población de gestantes programadas para cesárea, Cartagena, Colombia

\begin{tabular}{|c|c|c|}
\hline \multicolumn{3}{|c|}{ Características sociodemográficas, $n=326$} \\
\hline \multicolumn{3}{|c|}{ Variables Cuantitativas } \\
\hline \multicolumn{2}{|l|}{ Edad, $\mathrm{X} \pm \mathrm{SD}$} & $28,5 \pm 6,4$ \\
\hline \multicolumn{2}{|l|}{ Número de familiares en sala de espera, Me [RI] } & $2[1-2]$ \\
\hline \multicolumn{2}{|l|}{ Variables Cualitativas } & n (\%) [IC $95 \%]$ \\
\hline \multirow{4}{*}{ Rango de edad } & 13-19 años & $26(7,9)[5,5-11,4]$ \\
\hline & 20-29 años & $152(46,6)[41,2-52,0]$ \\
\hline & 30-39 años & $130(39,8)[34,1-45,2]$ \\
\hline & $>40$ años & $18(5,5)[3,5-8,5]$ \\
\hline \multirow{4}{*}{ Estado civil } & Soltera & $36(11,0)[8,0-14,9]$ \\
\hline & Casada & $153(46,9)[41,5-52,3]$ \\
\hline & Unión libre & $120(36,8)[31,7-42,1]$ \\
\hline & Separada & $17(5,21)[3,2-8,1]$ \\
\hline \multirow{2}{*}{ Actividad laboral } & Dentro de casa & $179(54,9)[49,4-60,2]$ \\
\hline & Fuera de casa & $147(45,0)[39,7-50,5]$ \\
\hline \multirow{3}{*}{ Número de embarazos } & Primero & $79(24,2)[19,9-27,1]$ \\
\hline & Segundo o tercero & $206(63,1)[57,8-68,2]$ \\
\hline & Cuarto y más & $41(12,5)[9,4-16,6]$ \\
\hline \multirow{2}{*}{ Número de abortos } & Ninguno & $219(67,1)[61,9-72,0]$ \\
\hline & Uno y más & $107(32,8)[27,9-38,0]$ \\
\hline \multirow{2}{*}{ Partos vaginales } & No & $254(77,9)[73,1-82,0]$ \\
\hline & Sí & $72(22,0)[17,9-26,9]$ \\
\hline \multirow{2}{*}{ Cesáreas previas } & No & $59(18,1)[14,3-22,6]$ \\
\hline & Sí & $267(81,9)[77,3-85,7]$ \\
\hline \multirow{2}{*}{ Antecedente de alguna cirugía } & No & $231(70,8)[65,7-75,5]$ \\
\hline & Sí & $95(29,1)[24,4-34,2]$ \\
\hline \multirow{2}{*}{ Antecedente de alguna complicación en el embarazo } & No & $270(82,8)[78,3-86,5]$ \\
\hline & Sí & $56(17,1)[13,4-21,6]$ \\
\hline \multirow{2}{*}{ Antecedente de dificultad para alcanzar el embarazo actual } & No & $302(92,7)[89,2-95,0]$ \\
\hline & Sí & $24(7,3)[5,0-10,72]$ \\
\hline
\end{tabular}

Fuente: creación propia

Se encontró Alpha de Cronbach 0,82 [IC $95 \%$ : 0,79-0,85] para la subescala de ansiedad y, 0,91 [IC $95 \%$ : 0,8960,929] para la subescala de necesidad de información.

De las variables exploradas solo el número de familiares en sala de espera se correlacionó sionificativamente con la AP $(\mathrm{p}=0,02)$, pero al interpretar el tamaño de la correlación se observa que es despreciable. Se encontró coeficiente de determinación $\left[\mathrm{r}^{2}\right]=0,016$, es decir, el 1,6\%. Ninguna de las variables se correlacionó con la necesidad de más información (Tabla 3). A su vez, la puntuación de la subescala de necesidad de información se correlacionó baja, positiva y sionificativamente con la de ansiedad, rho $=0,482$ [IC $95 \%$ : $0,394-0,561], \mathrm{p}<0,0001, \mathrm{r}^{2}=0,23$ que corresponde al $23 \%$. 
Tabla 2. Respuestas a cada uno de los ítems de la escala APAIS

\begin{tabular}{|c|c|c|c|c|c|c|}
\hline \multicolumn{7}{|c|}{ Amsterdam Preoperative Anxiety and Information Scale (APAIS), n= 326} \\
\hline & \multirow{3}{*}{ Ítems } & \multirow{2}{*}{$\begin{array}{c}\text { De ningún } \\
\text { modo } \\
1\end{array}$} & \multicolumn{3}{|c|}{ Intermedio } & \multirow{3}{*}{$\begin{array}{c}\text { Mucho } \\
5\end{array}$} \\
\hline & & & 2 & 3 & 4 & \\
\hline & & \multicolumn{4}{|c|}{ n (\%) [IC $95 \%]$} & \\
\hline 1 & Estoy preocupado sobre la anestesia & $\begin{array}{l}150(46,0) \\
{[40,6-51,4]}\end{array}$ & $\begin{array}{l}115(35,2) \\
{[30,2-40,6]}\end{array}$ & $\begin{array}{l}39(11,9) \\
{[8,8-15,9]}\end{array}$ & $\begin{array}{l}13(3,9) \\
{[2,3-6,7]}\end{array}$ & $\begin{array}{l}9(2,7) \\
{[1,4-5,1]}\end{array}$ \\
\hline 2 & La anestesia está en mi mente continuamente & $\begin{array}{l}153(46,9) \\
{[41,5-52,3]}\end{array}$ & $\begin{array}{l}117(35,8) \\
{[30,8-41,2]}\end{array}$ & $\begin{array}{l}33(10,1) \\
{[7,3-13,8]}\end{array}$ & $\begin{array}{l}13(3,9) \\
{[2,7-6,7]}\end{array}$ & $\begin{array}{l}10(3,0) \\
{[1,6-5,5]}\end{array}$ \\
\hline 3 & Quisiera saber todo lo posible sobre la anestesia & $\begin{array}{l}142(43,5) \\
{[38,2-48,9]}\end{array}$ & $\begin{array}{l}113(34,6) \\
{[29,7-39,9]}\end{array}$ & $\begin{array}{l}43(13,1) \\
{[9,9-17,3]}\end{array}$ & $\begin{array}{l}10(3,0) \\
{[1,6-5,5]}\end{array}$ & $\begin{array}{l}18(5,5) \\
{[3,5-8,5]}\end{array}$ \\
\hline 4 & Estoy preocupado sobre el procedimiento & $\begin{array}{l}149(45,7) \\
{[40,3-51,1]}\end{array}$ & $\begin{array}{l}110(33,7) \\
{[28,8-39,0]}\end{array}$ & $\begin{array}{l}40(12,2) \\
{[9,1-16,2]}\end{array}$ & $\begin{array}{l}19(5,8) \\
{[3,7-8,9]}\end{array}$ & $\begin{array}{l}8(2,4) \\
{[1,2-4,7]}\end{array}$ \\
\hline 5 & El procedimiento está en mi mente continuamente & $\begin{array}{l}148(45,4) \\
{[40,0-50,8]}\end{array}$ & $\begin{array}{l}107(32,8) \\
{[27,9-38,3]}\end{array}$ & $\begin{array}{l}39(11,9) \\
{[8,8-15,9]}\end{array}$ & $\begin{array}{l}17(5,2) \\
{[3,2-8,1]}\end{array}$ & $\begin{array}{l}15(4,6) \\
{[2,8-7,4]}\end{array}$ \\
\hline 6 & Quisiera saber todo lo posible sobre el procedimiento & $\begin{array}{l}151(46,3) \\
{[40,9-51,7]}\end{array}$ & $\begin{array}{l}94(28,8) \\
{[24,1-33,9]}\end{array}$ & $\begin{array}{l}41(12,5) \\
{[9,4-16,6]}\end{array}$ & $\begin{array}{l}14(4,2) \\
{[2,8-7,0]}\end{array}$ & $\begin{array}{l}26(7,8) \\
{[5,5-11,4]}\end{array}$ \\
\hline
\end{tabular}

Fuente: creación propia

Tabla 3. Ansiedad preoperatoria y necesidad de información

Correlación con las variables sociodemográficas. $n=326$

\begin{tabular}{|c|c|c|c|c|c|c|}
\hline \multirow{2}{*}{ Variables } & \multicolumn{3}{|c|}{ Ansiedad preoperatoria } & \multicolumn{3}{|c|}{ Necesidad de información } \\
\hline & rho & IC $95 \%$ & $\mathbf{p}$ & Rho & IC $95 \%$ & $\mathbf{P}$ \\
\hline Edad & $-0,004$ & $-0,113$ to 0,105 & 0,94 & $-0,034$ & $-0,142$ to 0,075 & 0,54 \\
\hline Embarazos & $-0,062$ & $-0,170$ to 0,047 & 0,26 & $-0,001$ & $-0,110$ to 0,107 & 0,98 \\
\hline Abortos & 0,005 & $-0,104$ to 0,113 & 0,93 & $-0,012$ & $-0,121$ to 0,097 & 0,82 \\
\hline Partos & $-0,075$ & $-0,182$ to 0,034 & 0,17 & $-0,065$ & $-0,172$ to 0,044 & 0,24 \\
\hline Cesáreas & 0,020 & $-0,089$ to 0,128 & 0,71 & 0,049 & $-0,060$ to 0,156 & 0,38 \\
\hline Cirugías previas & 0,018 & $-0,091$ to 0,126 & 0,75 & 0,021 & $-0,087$ to 0,130 & 0,70 \\
\hline Familiares en sala de espera & $-0,127$ & $-0,232$ to $-0,019$ & 0,02 & $-0,084$ & $-0,191$ to 0,025 & 0,13 \\
\hline
\end{tabular}

Fuente: creación propia

De las mujeres con AP el $60 \%$ informó tener necesidad de más información sobre la cirugía o anestesia, este aspecto se asoció con diez veces mayor frecuencia de la AP (Tabla 4). Los estados civiles unión libre y separación, con respecto a casadas, estuvieron asociados a mayor frecuencia de AP, OR: 3,7 [IC $95 \%: 1,5-9,2$ ], p $=0,004$ y OR: 4,4 [IC 95 \%: 1,0-19,3], $p=0,04$, respectivamente. Con respecto al grupo etario 20-29 años de edad, la adolescencia, tener entre 30-39 años y 40 o más años, no se asociaron siognificativamente con la AP.

10 
Tabla 4. Factores asociados con la ansiedad preoperatoria

\begin{tabular}{|c|c|c|c|}
\hline \multicolumn{4}{|c|}{$\begin{array}{l}\text { Regresión logística no ajustada } \\
\qquad n=326\end{array}$} \\
\hline \multirow{3}{*}{ Factores } & \multicolumn{2}{|c|}{$\begin{array}{l}\text { Ansiedad preoperatoria } \\
\qquad n=30(9,2 \%)\end{array}$} & \multirow{3}{*}{ OR (IC $95 \%)$} \\
\hline & Sí & No & \\
\hline & n (\%) & n (\%) & \\
\hline Necesidad de mucha más información & $18(60,0)$ & $12(40,0)$ & $10,9(4,54-26,36)$ \\
\hline Actividad laboral fuera de casa & $10(33,3)$ & $20(66,6)$ & $0,58(0,26-1,28)$ \\
\hline Dificultad para quedar en embarazo & $3(10,0)$ & $27(90,0)$ & $1,45(0,40-5,19)$ \\
\hline Antecedente complicación en embarazo actual & $8(26,7)$ & $22(73,3)$ & $1,87(0,79-4,46)$ \\
\hline Antecedente de cirugía previa & $10(33,3)$ & $20(66,6)$ & $1,24(0,55-2,76)$ \\
\hline Antecedente de cesárea & $24(80,0)$ & $6(20,0)$ & $0,87(0,33-2,23)$ \\
\hline Antecedente de parto vaginal & $4(13,3)$ & $26(86,6)$ & $0,51(0,17-1,52)$ \\
\hline Antecedente de aborto & $14(46,7)$ & $16(53,3)$ & $1,90(0,89-4,07)$ \\
\hline Primigestante & $8(26,7)$ & $22(73,3)$ & $1,15(0,49-2,70)$ \\
\hline Edad superior a 40 años & $1(3,3)$ & $29(96,6)$ & $0,56(0,07-4,40)$ \\
\hline Edad entre 30-39 años & $13(43,3)$ & $17(56,5)$ & $1,16(0,54-2,49)$ \\
\hline Edad entre $20-29$ años & $15(50,0)$ & $15(50,0)$ & $1,16(0,54-2,46)$ \\
\hline Adolescente (igual o menor 19 años) & $1(3,3)$ & $29(96,6)$ & $0,37(0,04-2,81)$ \\
\hline
\end{tabular}

Fuente: creación propia

Estar en primero, en segundo o tercer embarazo con respecto a cuatro o más gestaciones, tampoco estuvo significativamente asociado, OR: 1,5 [IC $95 \%$ : 0,5-4,4] y OR: 0,9 [IC $95 \%$ : 0,3-2,2], respectivamente. No se observaron factores asociados con menor presencia de la AP.

\section{DISCUSIÓN}

Entre los autores que introdujeron el término ansiedad se puede mencionar a Sigmund Freud, quien la usó como palabra técnica para enunciar una psicopatología que describe la respuesta adaptativa que prepara al organismo para reaccionar frente a situaciones estresantes o potencialmente nocivas ${ }^{(2)}$.

Diferentes condicionantes, especialmente etarios, educativos, sanitarios, culturales y sociales, pueden hacer que algunas personas perciban la cirugía o anestesia como peligrosa y por ello experimentan ansiedad $^{(3,6)}$. Se ha señalado que alounos neurotransmisores, especialmente la noradrenalina y la serotonina, con participación del sistema límbico, la amígdala y el hipocampo generan un aumento del factor liberador de corticotropina, incremento de la hormona adrenocorticotropa e hipersecreción de glucocorticoides, que se expresan con un aumento del tono muscular, taquicardia, sudoración y reacciones adversas cognitivas y psicológicas ${ }^{(1,4)}$. Dicho estado de ansiedad no solamente es antes de la cirugía, Hepp et al., (5) observaron en gestantes alemanas que la AP continuaba durante la cesárea e incluso después de finalizada la cirugía, reduciéndose en las horas siguientes.

La frecuencia esperada de la AP es diferente según la población, el tipo de intervención operatoria, la vía de administración anestésica y herramienta de medición ${ }^{(6,15-19)}$.

En general se considera que la prevalencia de la $\mathrm{AP}$ en poblaciones occidentales está entre el $60-80 \%$, aunque se ha anotado que el rango puede ser más amplio entre $11-80 \%{ }^{(6,10,11)}$. En el presente estudio, en embarazadas sanas, sin patologías materno fetales, sin condiciones obstétricas de urgencia o gravedad, programadas electivamente con cesárea, con consulta preanestésica realizada en días anteriores en el ámbito de la consulta externa y a recibir anestesia regional, se encontró una frecuencia de AP del 9,2 \% utilizando APAIS, la cifra más baja a la encontrada por Maheshwari e Ismail ${ }^{(20)}$ en gestantes de Pakistán, quienes con una escala visual análoga 
informaron que la $\mathrm{AP}$ entre las que recibirían anestesia regional fue de $51,8 \%$ y general $97,8 \%$. Mientras que en nuestro estudio se encontró una puntuación media de la subescala de ansiedad de 7,38 $\pm 3,45$. En una evaluación realizada en Eơipto ${ }^{(21)}$, también con escala APAIS en mujeres con preeclampsia, encontraron una puntuación superior, no informaron sobre la frecuencia de la $\mathrm{AP}$ e indicaron que realizar premedicación con midazoIam permitía reducir la puntuación del APAIS de 17,1 \pm 3,1 , antes de pasar la paciente a la sala de ciruoía, al 11,9 $\pm 1,9$ a los cinco minutos de aplicada la premedicación. Detectar la AP obliga a realizar un seguimiento e intervención terapéutica. No se encontraron otros estudios con APAIS en mujeres que fueron sometidas a cesárea.

Teena y Akanksha ${ }^{(22)}$, en una población hindú, encontraron con la escala STATE que el $55 \%$ de 200 mujeres que serían sometidas a cesárea presentaban AP. Aparte de esto anotaron que la frecuencia fue sionificativamente mayor en las mujeres sometidas a cesárea de emergencia con respecto a la cesárea electiva; 70 \% y $40 \%$, respectivamente. Usualmente las gestantes que van a ser sometidas a cesárea le temen a la anestesia, al procedimiento operatorio, a morir, a presentar complicaciones, a la cicatriz quirúrogica y al estado no saludable del neonato, todo lo cual se traduce en $\mathrm{AP}{ }^{(23)}$.

Aunque se ha señalado que las pacientes obstétricas tienen una mayor prevalencia de la $\mathrm{AP}$ al compararlas con la población sometida a otros tipos de cirugías ${ }^{(19}$. ${ }^{22)}$, encontramos que la frecuencia de la AP en este estudio de gestantes del caribe de Colombia fue inferior al 62,0 \% reportado en Pakistán ${ }^{(19)}, 76,7$ \% en Sri Lanka (18) y 70,3 \% en Etiopía ${ }^{(24)}$; en estos estudios las poblaciones eran heterogéneas, sometidas a diferentes tipos de cirugías, con diversas morbilidades, valoradas con diferentes herramientas de medición y de mayor edad que las gestantes que hemos considerado. Las incluidas en el presente estudio tenían rango intercuartílico 22-34 años y el 80 \% estaba entre 20-39 años de edad. Esta última variable puede explicar en algo las diferencias observadas, ya que la AP tiene una asociación con la edad, la frecuencia puede ser menor en la mediana edad si se compara con la observada en las edades mayores al analizar amplios grupos etarios ${ }^{(6)}$.

La menor frecuencia encontrada también se puede explicar por el grupo de estudio: embarazadas sanas intervenidas por tener antecedente de una o más cesáreas o, por el hallazgo clínico de condiciones obstétricas relativamente frecuentes y no graves. No se identificaron estudios similares en otras gestantes colombianas, sanas o enfermas.

Se observó que la puntuación de la subescala de ansiedad se correlaciona negativamente y con significancia estadística con el número de familiares en la sala de espera, aunque el tamaño del coeficiente de correlación fue muy bajo, clasificado en niveles despreciables con la escala seleccionada para la interpretación. Varias escalas han sido propuestas, Schober et aI., ${ }^{(25)}$ Señalan que algunos puntos de corte pueden ser arbitrarios e inconsistentes y se deben juiciosamente considerar al interpretar las cifras. Además, ellos apuntan como ejemplo que un coeficiente de correlación entre 0,10-0,39 sería una correlación débil. Hacen notar que existe mayor consenso entre los autores que $<0,1$ es despreciable y $>0,9$ es muy fuerte, las dificultades están en los cortes intermedios. No obstante, el número de familiares en la sala de espera solo explica el 1,6\% de la varianza. Al quedar más del 80 \% sin explicación indica que otros factores deben tener importante presencia.

Aunque haya diferencias en la interpretación de los haIlazgo o cálculos estadísticos, se puede especular que, por ser la familia el núcleo básico social, al menos en nuestro entorno, cumple un importante rol de protección y amparo para sus integrantes, especialmente, cuando se encuentran recibiendo atención en salud ${ }^{(26)}$.

Fuchs-Tarlorysky et al. ${ }^{(27)}$, han señalado que la movilización o el mutuo apoyo entre los integrantes de la familia para el cuidado o acompañamiento puede ser factor de protección para la depresión en mujeres no gestantes hospitalizadas.

No se identificaron otros estudios en embarazadas que describieran la correlación observada, ya que la cuantificación de los familiares en la sala de espera no estaba entre las variables sociodemográficas. Estudios mucho más grandes, multicéntricos y multiétnicos permitirán definir en mejor forma la adecuada correlación entre el número de familiares en la sala de espera y la $\mathrm{AP}$ en gestantes.

También se observó que estar en separada o en unión libre, con respecto a estar casada, fueron factores que se asociaron a cuatro veces más presencia de AP. No se identificaron hallazos similares en otros estudios. Hacen falta evaluaciones desde el punto de vista sociológico y psicológico para explicar dicho hallazgo y dimensionarlo en su esencia. Se hace necesario incrementar la mirada psicosocial de los aspectos biomédicos en general, específicamente, en la AP. 
El tamaño del coeficiente de correlación entre la necesidad de información y la AP fue observado bajo [rho $=0,48]$, con la escala de interpretación seleccionada ${ }^{(14)}$, mientras que Schober et al. ${ }^{(25)}$, con el enfoque convencional de evaluación, la interpretó moderada [rho: 0,400,69]. La necesidad de información explica el $23 \%$ de la variación de la AP en las gestantes sanas que fueron estudiadas. Con un cálculo del tamaño de la muestra más adecuadamente estimado, con una mayor población como universo de estudio y atendidas en varias instituciones sanitarias se puede llegar a definir de mejor forma los alcances de la correlación.

El 60 \% de las gestantes a las cuales se les identificó la $\mathrm{AP}$, afirmaron tener necesidad de mucha más información sobre la cirugía o anestesia. A la vez, la necesidad de mucha más información fue el único factor que se encontró asociado significativamente a mayor frecuencia de AP. La completa y amplia información sobre el procedimiento operatorio y el acto anestésico, sumada a la eficiente comunicación entre el paciente y profesional de la salud, debe generar satisfacción, un ambiente clínico confortable y la percepción del cuidado médico humanitario.

Al respecto, Hobson et aI. ${ }^{(28)}$, indican que reducir la $\mathrm{AP}$ se acompaña de un incremento en la satisfacción materna posoperatoria después de la cesárea electiva. Además, se ha señalado, aunque en pacientes no obstétricas, que la satisfacción con la anestesia recibida está influenciada por la adecuada información suministrada, la cual se puede hacer de modo oral, escrita, fotográfica o audiovisual, acompañada o no con música ${ }^{(29)}$.

Al respecto, Eley et aI. ${ }^{(30)}$, en un estudio en gestantes australianas, observó que no existían diferencias sionificativas en cuanto a la frecuencia de la AP y el grado de satisfacción materna, cuando un ơrupo recibió la información sobre la cirugía o anestesia de forma tradicional y otro, tanto la información tradicional como la aportada por un video educativo. Concluyen que agregar información en video no reduce la AP, no obstante, todas las herramientas pueden ser válidas para tratar que las gestantes que serán sometidas a cesárea tengan poca a ninguna necesidad de más información ${ }^{(29,30)}$.

En el presente estudio el $72,1 \%$ tenían poca a ninguna necesidad de información, lo que indica que se ameritan más y mejores esfuerzos educativos o informativos tanto en la valoración y consejería obstétrica, como en la consulta anestésica preoperatoria.
El estudio tiene las limitaciones propias de los diseños transversales: no establece causalidad sino asociación estadística. Los resultados obtenidos son propios del grupo de estudio y no necesariamente deben ser ampliamente extrapolados, ya que responden a un tamaño muestral de una institución de salud. No tuvo entre sus focos principales explorar la asociación de la AP con aspectos clínicos, metabólicos o hemodinámicos maternos intraoperatorios, posoperatorios o neonatales $y$, tampoco con el grado de satisfacción en la atención obstétrica.

Tiene como fortaleza haber sido realizado con APAIS, una escala internacionalmente propuesta para identificar la AP, herramienta corta, fácilmente comprensible que permite la valoración desde la misma mirada del paciente que se va a someter a una ciruoía. Wyatt et al., (11), anotan que los niveles de ansiedad son difíciles de valorar y no existe una prueba específica de medición, añaden que la ansiedad en la psicología humana es usualmente basada en la introspección y en el autorreporte, utilizando la escala visual análoga u otras.

Las evaluaciones objetivas son incluso indirectas al valorar la actividad neuroendocrina, midiendo el estado hemodinámico, el cortisol sérico o en saliva y las catecolaminas urinarias ${ }^{(5,6)}$. Con el fin de obtener cifras de la AP en la población obstétrica sana, se estudiaron mujeres sin enfermedades obstétricas, maternas o fetales, ya que estas condiciones pueden ser factores que incrementan la presencia de la AP. El presente estudio es de los primeros realizados en gestantes latinoamericanas con escala APAIS, se ameritan muchos más.

Aporta tres aspectos: primero, identificó una frecuencia inferior al $10 \%$ de la AP en un grupo de gestantes sanas de una institución de salud, minutos antes de la cesárea. Segundo, refuerza lo señalado previamente por diversos autores ${ }^{(6)}$, acerca de la importancia de la adecuada información sobre la cirugía o anestesia para reducir la frecuencia de la AP. Tercero, hace un primer señalamiento, que a mayor presencia de familiares en la sala de espera, pude llegar a existir una menor AP.

Aunque la correlación fue estadísticamente significativa, el tamaño del coeficiente de correlación fue muy bajo, se interpreta como despreciable o débil según la escala de medición. Se debe anotar que una correlación estadísticamente significativa no se debe confundir con una correlación clínicamente relevante ${ }^{(25)}$. 
Los hallazgos del estudio permiten recomendar a los profesionales de la salud que van a realizar intervenciones operatorias o actos anestésicos obstétricos, la importancia de brindar amplia y suficiente información. Hasta que datos diferentes estén disponibles, pese a las cifras estadísticas no apreciables, las instituciones de atención obstétrica deben fomentar la presencia de familiares acompañantes en las salas de espera, en vez de limitarlos o controlarlos, por lo menos por razones humanitarias. Ya que la AP está asociada con mayores exigencias sanitarias o quirúroicas ${ }^{(3,5)}$. Todas las intervenciones médicas $y$ administrativas que contribuyen a reducirla se deben considerar.

Desde la psicobiología se debe estudiar la interrelación de la AP con el optimismo, la susceptibilidad y la capacidad para abordar condiciones estresantes, sin dejar de lado el aspecto sociocultural ${ }^{(6,10,11)}$. En 1985 la OMS ${ }^{(31)}$ recomendó que en la atención a gestantes se debe considerar como fundamentales los factores sociales, emocionales y psicológicos, todos ellos hacen parte de la atención perinatal de calidad.

\section{CONCLUSIÓN}

Se observó que el 9,2\% de un grupo de gestantes sanas, residentes en el caribe colombiano, y próximas a ser intervenidas con cesárea, presentaron AP. La necesidad de mucha más información sobre la cirugía o anestesia se asoció a diez veces mayor presencia de la AP. El número de familiares en la sala de espera se correlacionó negativamente con la AP, aunque el tamaño del coeficiente de correlación fue interpretado como despreciable.

\section{AGRADECIMIENTOS}

A las directivas de la Clínica Santa Cruz de Bocagrande Cartagena, Colombia. A las auxiliares de enfermería Rosiris Pereira y Genolvis Buelvas, quienes realizaron el trabajo de campo identificando la población y aplicando la herramienta de estudio.

\section{FINANCIACIÓN}

Estudio apoyado por la vicerrectoría de investigaciones de la Universidad de Cartagena, Colombia. Resolución
N. 00961 del 2017: trámite para la obtención de recursos financieros en apoyo y sostenibilidad de grupos de investigación avalados por la Universidad de Cartagena; acta de compromiso N. ${ }^{\circ}$ 126-2017 entre la Universidad de Cartagena y el Grupo de Investigación Salud de la Mujer. Los investigadores no recibieron honorarios específicamente por adelantar la investiogación.

\section{PRESENTACIÓN COMO CONFERENCIA}

Una versión parcial del artículo fue presentada por FQG en la sección de presentación oral deI XXII World Congress Of Gynecology And Obstetric de la Federación Internacional de Ginecología y Obstetricia, Río de Janeiro: Brasil, 2018.

\section{CONFLICTOS DE INTERESES}

Ninguno por declarar.

\section{REFERENCIAS BIBLIOGRÁFICAS}

1. Boyer P. Do anxiety and depression have a common pathophysiological mechanism? Acta Psychiatr Scand Suppl. 2000;(406):24-9.

2. Sierra JC, Ortega $\nabla$, Zubeidat I. Ansiedad, angustia y estrés: tres conceptos a diferenciar. Rev Mal-Estar Subj. 2003;3(1):10-59.

3. Hernández Hernández AI, López Bascope AJ, Guzmán Sánchez JA. Nivel de ansiedad e información preoperatoria en pacientes programados para ciruogía. Un estudio transversal descriptivo. Acta Med. 2016;14(1):6-11.

4. Olofsdotter S. Anxiety among adolescents: measurement, clinical characteristics, and influences of parenting and genetics. [Doctoral dissertation]. UppsaIa: Uppsala Universitet; 2017.

5. Hepp P, Hagenbeck C, Burghardt B, Jaeger B, Wolf OT, Fehm T, et al. Measuring the course of anxiety in women giving birth by caesarean section: a prospective study. BMC pregnancy and childbirth. 2016;16:113. DOI 10.1186/s12884-016-0906-z.

6. Woldegerima YB, Fitwi GL, Yimer HT, Hailekiros AG. Prevalence and factors associated with preoperative 
anxiety among elective surgical patients at University of Gondar Hospital. Gondar, Northwest Ethiopia, 2017. A cross-sectional study. IJSO. 2018;10:21-9. DOI 10.1016/j.ijso.2017.11.001.

7. Vergara-Romero M, Morales-Asencio JM, MoralesFernández A, Canca-Sánchez JC, Rivas-Ruiz F, Reinaldo-Lapuerta JA. Validation of the Spanish version of the Amsterdam Preoperative Anxiety and Information Scale (APAIS). Health Qual Life Outcomes. 2017;15(1):120

8. Colombia. Ministerio de Salud y Protección Social de Colombia. Atlas de Variaciones Geográficas en Salud de Colombia. 2015 - Estudio piloto - Resultados de partos por cesárea [internet]. [Consultado 2019 jul 9]. Disponible: http://cort.as/-L7vQ

9. Organización Mundial de la Salud. Declaración de la OMS sobre tasas de cesárea [internet]. [Consultado 2019 jul 9]. Disponible: http://cort.as/-L7vd

10. Chen HH, Lai JC, Hwang SJ, Huang N, Chou YJ, Chien LY. Understanding the relationship between cesarean birth and stress, anxiety, and depression after childbirth: A nationwide cohort study. Birth. 2017;44(4):36976. DOI 10.1111/birt.12295.

11. Wyatt SS, Jones DA, Peach MJ, Gurrin L. Anxiety in patients having caesarean section under regional anaesthesia: a questionnaire and pilot study. Int J Obstet Anesth. 2001;10(4):278-83. DOI 10.1054/ ijoa.2001.0866.

12. World Medical Association. Declaración de Helsinki de la AMM - Principios éticos para las investigaciones médicas en seres humanos [internet]. [Consultado 2019 jul 9]. Disponible: http://cort.as/-L7w2

13. Colombia. Ministerio de Salud de Colombia. Resolución número 8430 DE 1993. Normas científicas, técnicas y administrativas para la investigación en salud [internet]. [Consultado 2019 jul 9]. Disponible: http:// cort.as/-IVzd

14. Mukaka MM. A guide to appropriate use of correlation coefficient in medical research. Malawi Med J. 2012;24(3):69-71.

15. Yilmaz M, Sezer H, Gürler H, Bekar M. Predictors of preoperative anxiety in surgical inpatients. J Clin Nurs. 2012;21(7-8):956-64. DOI 10.1111/j.13652702.2011.03799.x.

16. Wilson CJ, Mitchelson AJ, Tzeng TH, El-Othmani MM, Saleh J, Vasder S, et al. Caring for the surgically anxious patient: a review of the interventions and a guide to optimizing surgical outcomes. Am J Surg. 2016;212(1):151-9. DOI 10.1016/j.amjsurg.2015.03.023.

17. Erkilic E, Kesimci E, Soykut C, Doger C, Gumus T, Kanbak O. Factors associated with preoperative anxiety levels of Turkish surgical patients: from a single center in Ankara. Patient Prefer Adherence. 2017;11:291-6. DOI 10.2147/PPA.S127342.

18. Matthias AT, Samarasekera DN. Preoperative anxiety in surgical patients - experience of a single unit. Acta anaesthesiol Taiwan. 2012;50(1):3-6. DOI 10.1016/j. aat.2012.02.004.

19. Jafar MF, Khan FA. Frequency of preoperative anxiety in Pakistani surgical patients. J Pak Med Assoc. 2009;59(6):359-63.

20. Maheshwari D, Ismail S. Preoperative anxiety in patients selecting either general or regional anesthesia for elective cesarean section. J Anaesthesiol Clin Pharmacol. 2015;31(2):196-200. DOI 10.4103/09709185.155148 .

21. Mokhtar AM, Elsakka AI, Ali HM. Premedication with midazolam prior to cesarean delivery in preeclamptic parturients: A randomized controlled trial. Anesth Essays Res. 2016;10(3):631-6. DOI 10.4103/0259-1162.191117.

22. Teena Bansal DA, Akanksha Joon MD. A comparative study to assess preoperative anxiety in obstetric patients undergoing elective or emergency cesarean section. Anaesth Crit Care Pain Med. 2017;21(1):25-30.

23. Sahin T, Gulec E, Sarac Ahrazoglu M, Tetiker S. Association between preoperative maternal anxiety and neonatal outcomes: a prospective observational study. J Clin Anesth. 2016;33:123-6. DOI 10.1016/j.jclinane.2016.03.022

24. Nigussie S, Belachew T, Wolancho W. Predictors of preoperative anxiety among surgical patients in Jimma University Specialized Teaching Hospital, South Western Ethiopia. BMC Surg. 2014;14:67. DOI 10.1186/1471-2482-14-67.

25. Schober P, Boer C, Schwarte LA. Correlation coefficients: appropriate use and interpretation. Anesth Analg. 2018;126(5):1763-8. DOI 10.1213/ ANE.0000000000002864.

26. Zarei M, Keyvan M, Hashemizadeh H. Assessing the Level of Stress and Anxiety in Family Members of Patients Hospitalized in the Special Care Units. Int. J. Rev. Life. Sci. 2015;5(11):118-22. 
27. Fuchs-Tarlovsky $\nabla$, Bejarano M, Álvarez K, Godoy M, Fernández NC. Efecto de la Presencia de los Familiares sobre la Depresión en Mujeres Hospitalizadas con Cáncer. Rev Venez Oncol. 2013;25(3):190-5.

28. Hobson JA, Slade P, Wrench IJ, Power L. Preoperative anxiety and postoperative satisfaction in women undergoing elective caesarean section. Int J Obstet Anesth. 2006;15(1):18-23. DOI 10.1016/j. ijoa.2005.05.008.

29. Gómez-Urquiza JL, Hueso-Montoro C, UrquizaOlmo J, Ibarrondo-Crespo R, González-Jiménez E,
Schmidt-Riovalle J. A randomized controlled trial of the effect of a photographic display with and without music on pre-operative anxiety. J Adr Nurs. 2016;72(7):1666-76. DOI 10.1111/jan.12937.

30. Eley VA, Searles T, Donovan K, Walters E. Effect of an anaesthesia information video on preoperative maternal anxiety and postoperative satisfaction in elective caesarean section: a prospective randomised trial. Anaesth Intensive Care. 2013;41(6):774-81. DOI 10.1177/0310057X1304100613.

31. World Health Organization. Appropriate technology for birth. Lancet. 1985; 326(8452):436-437. 